PROCEEDINGS OF THE

AMERICAN MATHEMATICAL SOCIETY

Volume 125, Number 12, December 1997, Pages 3721-3723

S 0002-9939(97)03994-4

\title{
INDEPENDENCE AND DETERMINATION OF PROBABILITIES
}

\author{
ZHIQIANG CHEN, HERMAN RUBIN, AND RICHARD A. VITALE
}

(Communicated by Stanley Sawyer)

\begin{abstract}
A non-atomic probability measure is determined by its specification of independent events. We give two proofs and related results.
\end{abstract}

\section{INTRODUCTION}

Suppose that $P$ and $Q$ are two probability measures on the same measurable space. We say that they have identical independent events if, for any pair of events $A$ and $B, P(A B)=P(A) P(B)$ if and only if $Q(A B)=Q(A) Q(B)$. Two probability measures having the same independent events may be quite unrelated, as, for example, in simple point mass examples. A natural question then arises: "What properties, if any, are shared by two probability measures with identical independent events?" In this note, we show somewhat unexpectedly that the probability measures must actually coincide when point masses are disallowed, or, more precisely, in the nonatomic case.

That the independence structure of a probability measure can determine its actual values appears to be a novel idea. In the next sections, we give the formal statement with two proofs and two related results.

\section{Statement And First PRoOF}

We assume throughout probability measures $P$ and $Q$ on a fixed measurable space $(\Omega, \mathcal{F})$. Recall that an event $A \in \mathcal{F}$ is an atom of $P$ if $P(A)>0$ and if for any $B \subseteq A$, either $P(B)=P(A)$ or $P(B)=0$. $P$ is nonatomic if it has no atoms.

Our main result is as follows.

Theorem 1. Suppose that $P$ and $Q$ are two probability measures at least one of which is nonatomic. If they have identical independent events, then they coincide.

The proof is based on two lemmas.

Lemma 1. If $P$ is a nonatomic measure with $P(A)>0$, then there are events $B \subseteq A, C \subseteq A$ such that $P(B C)=P(B) P(C) \neq 0$.

Received by the editors March 12, 1996 and, in revised form, July 1, 1996.

1991 Mathematics Subject Classification. Primary 60A99; Secondary 28 A99.

Key words and phrases. Determining conditions, independence, Lyapunov Convexity Theorem, non-atomic measure, stochastic independence.

The first author was supported in part by a grant from the Center for Research, School of Science and Health, William Paterson College.

The third author was supported in part by ONR Grant N00014-90-J-1641.

(C) 1997 American Mathematical Society 
Proof. Setting $p=P(A)$, we can find three mutually exclusive subevents $A_{1}, A_{2}, A_{3}$ of $A$ with $P\left(A_{1}\right)=P\left(A_{2}\right)=p / 2-p^{2} / 4$ and $P\left(A_{3}\right)=p^{2} / 4$ (e.g. [2, p. 83], [6, p. 101]). Then $B=A_{1} \cup A_{3}$ and $C=A_{2} \cup A_{3}$ provide a solution.

Lemma 2. If $P$ is nonatomic and if $P$ and $Q$ have identical independent events, then $P$ is absolutely continuous with respect to $Q$.

Proof. Suppose that $Q(A)=0$. It follows a fortiori that $A$ is independent of itself under $Q$ and hence by assumption under $P$. Consequently $P(A)=0$ or 1 . We rule out the latter by observing that, if it did hold, then $P$ being non-atomic would imply the existence of $B \subset A$ such that $0<P(B)<1$. But by the same reasoning as before, $Q(B)=0$ forces $P(B)=0$.

Proof of Theorem 1. Suppose that $P$ is nonatomic. Using Lemma 2, let $f$ be the Radon-Nikodym derivative $d P / d Q$. We show that $f \leq 1$ and hence $f \equiv 1 Q$-a.s. It is enough to verify that, for each $\delta>0, Q$ assigns zero probability to each event $A_{\delta}=\{1+\delta<f<1+2 \delta\}$ since $\{1<f\}$ can be written as a countable union of such events. Suppose, to the contrary, that $Q\left(A_{\delta}\right)>0$. Then $P\left(A_{\delta}\right)>0(f>1+\delta$ on $\left.A_{\delta}\right)$, and by Lemma 1 there are $B, C \subseteq A_{\delta}$ with $P(B \cap C)=P(B) P(C) \neq 0$. Lemma 2 implies that $Q(B \cap C)=Q(B) Q(C) \neq 0$. But then $(1+2 \delta) Q(B) Q(C)=$ $(1+2 \delta) Q(B C) \geq \int_{B C} f d Q=P(B \cap C)=P(B) P(C)=\int_{B} f d Q \cdot \int_{C} f d Q \geq$ $(1+\delta)^{2} Q(B) Q(C)$, which implies $(1+2 \delta) \geq(1+\delta)^{2}$, contradicting $\delta>0$.

Corollary 1. Suppose that $P$ and $Q$ are two probability measures at least one of which is nonatomic. If they have identical mutually favorable events in the sense that, for any events $A$ and $B$,

$$
P(A B) \geq P(A) P(B) \Longleftrightarrow Q(A B) \geq Q(A) Q(B),
$$

then $P$ and $Q$ coincide.

Proof. It is clear by complementation that (1) implies that $P$ and $Q$ have identical mutually unfavorable events as well. It follows easily that $P$ and $Q$ have identical independent events, and Theorem 1 then applies.

\section{SECOND PROOF}

An alternate proof of Theorem 1 uses the Lyapunov Convexity Theorem (e.g. [1], [3], [4], [5], [7]):

Theorem 2. The range of a nonatomic vector measure is compact convex.

We also use the following lemma, which can be verified by direct computation.

Lemma 3. For any probability measure $P$ and events $A$ and $B$, if both $P(A)$ and $P(B)$ lie in $(0,1)$, then the three events $A, B$, and $A \Delta B$ are pairwise independent iff $P(A)=P(B)=2 P(A B)=1 / 2$.

Second proof of Theorem 1. Without loss of generality, let $P$ be nonatomic. We show first that for any event $A$, if $P(A)=1 / 2$, then $Q(A)=1 / 2$ : there are $A_{1} \subseteq A$ and $A_{2} \subseteq A^{c}$ with $P\left(A_{1}\right)=P\left(A_{2}\right)=1 / 4$. Let $\tilde{A}=A_{1} \cup A_{2}$. It is immediate that $A, \overline{\tilde{A}}, A \Delta \tilde{A}$ are pairwise independent under $P$ and by assumption also under $Q$. Hence $Q(A)=1 / 2$ by Lemma 3 .

Now suppose $A$ is given with $P(A) \neq 1 / 2$; without loss of generality $P(A)>1 / 2$. Because $P$ is nonatomic, the vector measure $(P, Q)$ is also nonatomic. Since $(0,0)=$ 
$(P(\phi), Q(\phi))$, the Lyapunov Convexity Theorem ensures that for each $0<t<1$, there is an event $B$ such that $(P(B), Q(B))=t(P(A), Q(A))$. In particular, for $t=[2 P(A)]^{-1}$, we have $P(B)=1 / 2$. From the first part of the proof it follows that $Q(B)=1 / 2$ and in turn $Q(A)=P(A)$.

Elements of the proof provide also the following characterization.

Corollary 2. Let $P$ and $Q$ be two probability measures at least one of which is nonatomic. If

$$
P(A)=1 / 2 \Longleftrightarrow Q(A)=1 / 2
$$

then $P=Q$.

\section{REMARK}

In view of current activity in Bayesian and Markovian models with specified dependency structures, one might ask whether there are similar results to the foregoing under some form of conditioning.

\section{REFERENCES}

[1] Diestel, J. and Uhl, Jr., J.J. (1977). Vector Measures. Amer. Math. Soc. Surveys 13. MR 56:12216

[2] Dudley, R.M. (1989). Real Analysis and Probability. Wadsworth and Brooks/Cole, Pacific Grove. MR 91g:60001

[3] Dvoretzky, A., Wald, A., and Wolfowitz, J. (1951). Relations among certain ranges of vector measures. Pac. J. Math. 1, 59-74. MR 13:331f

[4] Kluvánek, I. (1980). Applications of vector measures. In Integration, Topology, and Geometry of Linear Spaces. Contemporary Mathematics 2, Amer. Math. Soc. (W.H. Graves, R.L. Davis, F.B. Wright, eds.). MR 82m:28019

[5] Lehmann, E.L. (1959). Testing Statistical Hypotheses. Wiley, New York. MR 21:6654

[6] Loève, M. (1963). Probability Theory, I (third edition). Van Nostrand, New York. MR 34:3596

[7] Lyapunov, A. (1940). Sur les fonctions-vecteurs completèment additives. Izvestia Akad. Nauk SSSR Ser. Mat. 4 (Russian, French summary) 465-478.

Department of Mathematics, William Paterson College, Wayne, New Jersey 07470

E-mail address: z_chen@smtplink.wilpaterson.edu

Department of Statistics, Purdue University, West Lafayette, Indiana 47907

E-mail address: hrubin@stat.purdue.edu

Department of Statistics, University of Connecticut, Storrs, Connecticut 06269

E-mail address: rvitale@uconnvm.uconn.edu 